
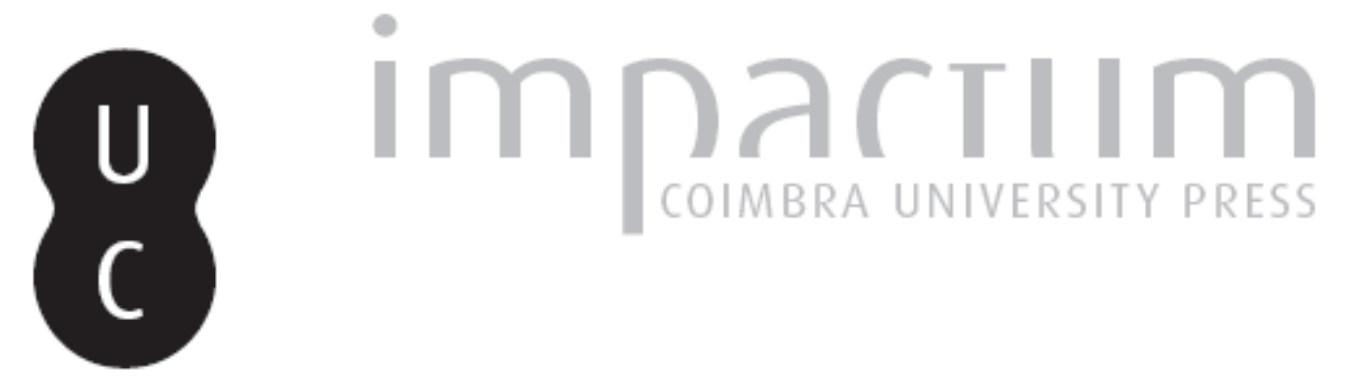

\title{
La ricezione dell'ode II Cinque Maggio nel Portogallo e nel Brasile del XIX secolo
}

Autor(es): $\quad$ Fiorentino, Viola

Publicado por: Imprensa da Universidade de Coimbra

URL persistente:

URI:http://hdl.handle.net/10316.2/42549

DOI: $\quad$ DOI:https://doi.org/10.14195/0870-8584_6_7

Accessed : $\quad$ 26-Apr-2023 00:51:50

A navegação consulta e descarregamento dos títulos inseridos nas Bibliotecas Digitais UC Digitalis, UC Pombalina e UC Impactum, pressupõem a aceitação plena e sem reservas dos Termos e Condições de Uso destas Bibliotecas Digitais, disponíveis em https://digitalis.uc.pt/pt-pt/termos.

Conforme exposto nos referidos Termos e Condições de Uso, o descarregamento de títulos de acesso restrito requer uma licença válida de autorização devendo o utilizador aceder ao(s) documento(s) a partir de um endereço de IP da instituição detentora da supramencionada licença.

Ao utilizador é apenas permitido o descarregamento para uso pessoal, pelo que o emprego do(s) título(s) descarregado(s) para outro fim, designadamente comercial, carece de autorização do respetivo autor ou editor da obra.

Na medida em que todas as obras da UC Digitalis se encontram protegidas pelo Código do Direito de Autor e Direitos Conexos e demais legislação aplicável, toda a cópia, parcial ou total, deste documento, nos casos em que é legalmente admitida, deverá conter ou fazer-se acompanhar por este aviso.

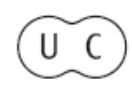




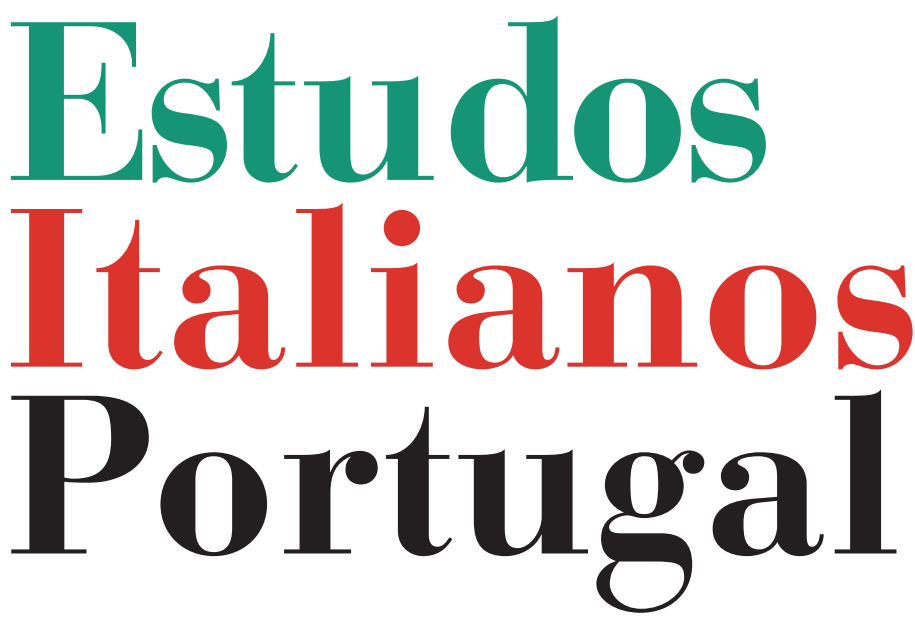

Instituto

Italiano

de Cultura

de Lisboa

Nova Série

$\mathrm{N}^{\circ} 6$ 


\section{Viola Fiorentino*}

Era il 16 luglio 1821 quando Alessandro Manzoni, nel giardino della sua residenza a Brusuglio, apprese la notizia della morte di Napoleone, riportata dalla Gazzetta di Milano. Profondamente commosso dall'inatteso evento, lo scrittore si immerse subito nella stesura dell'ode, che scrisse di getto e portò a termine con una rapidità inaudita, nel giro di appena tre o quattro giorni.

Manzoni ci regala così un componimento che vuole sì rendere omaggio all'imperatore appena scomparso, ma che al tempo stesso è anche occasione per una più generale riflessione di carattere religioso ed esistenziale. Come è possibile apprezzare scorrendo i versi incalzanti della poesia, che riportiamo di seguito in appendice, nel Cinque maggio l'«uom fatale» è colto nel suo insieme. Un personaggio grandioso, una genialità bellica, ma pur sempre un uomo che, in quanto tale, è soggetto alla dinamica ineluttabile del corso della vita. Da questa descrizione caleidoscopica e sfaccettata trapela anche lo stato d'animo complesso, quasi contraddittorio, che

* Viola Fiorentino (Pisa, 1984) frequenta la Laurea Specialistica in Linguistica Teorica Applicata dell'Università di Pisa. Ha lavorato presso l'Istituto di Studi Italiani dell'Università di Coimbra nell'ambito del programma Erasmus Placement. Si è occupata di storia della comunità ebraica di origine portoghese di Livorno, in particolare sotto il profilo linguistico-letterario. 
dovette guidare la penna dell'autore che, lungi dall'essere un sostenitore bonapartista - e Aprile $1814^{1}$ ne è un esempio lampante -, non poteva esimersi dal provare, nonostante le riserve morali e religiose, una sostanziale ammirazione per questo grandioso personaggio.

Dal punto di vista formale, Il cinque maggio è un'ode composta da diciotto strofe di sei versi settenari ciascuna, di cui il primo, il terzo e il quinto sdruccioli, il secondo e il quarto piani e rimanti, il sesto tronco e in rima con i versi finali delle altre strofe. Quest'alternanza di versi sdruccioli, piani e tronchi, insieme a una sintassi che accosta periodi fortemente paratattici a larghe volute ipotattiche, crea un ritmo rapido e concitato, che rallenta solo nei momenti di riflessione e di più commossa eloquenza.

Tradotta in tedesco da Goethe ${ }^{2}$, invidiata da Lamartine, che in una lettera al suo amico De Virieu scrisse significativamente, "Je voudrais l'avoir faite"3, giudicata intraducibile da Longfellow, citata da scrittori del calibro di Hugo, Béranger, Reboul, Musset, Delavigne e Méry, l’ode di Manzoni raggiunse un successo strepitoso in tutta l'Europa del XIX secolo ${ }^{4}$, compiendosi in breve tempo la profezia dell'autore:

\footnotetext{
${ }^{1}$ Nella poesia, composta alla fine dell'occupazione francese della Penisola, Manzoni lancia un'invettiva alle truppe napoleoniche che, invece di liberare la penisola, vi portarono di fatto una nuova tirannide.

${ }^{2}$ La traduzione del Goethe, la prima in ordine cronologico, venne letta alla corte di Weimar l'8 Agosto 1822 dallo stesso, per essere poi pubblicata verso la fine del medesimo anno nella rivista Uber Künst und Alterthum.

${ }^{3}$ Angelo De Gubernatis, Alessandro Manzoni. Studio biografico, Firenze, Successori Le Monnier, 1879, p. 209.

${ }^{4}$ Vitorino Nemésio riferisce persino che Herculano prese come modello il componimento di Manzoni per lodare il suo collega Soares de Passos: "O seu Camóes é uma cousa admirável, e com leves retoques eu não hesitaria em pô-lo a par do Cinque maggio de Manzoni”, A mocidade de Herculano, I, Lisboa, Bertrand, 1978, p.287.
} 
e scioglie all'urna un cantico

che forse non morrà. ${ }^{5}$

La notorietà che riscosse il componimento fu tale da sorpassare se possibile quella dei Promessi sposi. Difatti, in Portogallo come in Brasile, Manzoni era inaspettatamente conosciuto, e magari più che come poeta e autore teatrale, per il celebre romanzo storico che costituisce una delle colonne portanti della nostra letteratura. A conferma di quanto detto, Vitorino Nemésio ${ }^{6}$ riporta che la moda di Manzoni in Portogallo raggiunse probabilmente il suo apice nel 1841, in seguito alla pubblicazione delle sue Osservazioni sulla morale cattolica e dei Promessi sposi sulla Revista Universal Lisbonense, ma che la fama dello scrittore era giunta nel paese già da tempo, proprio grazie alle sue odi civili e in particolare a Il cinque maggio, che veniva recitato con commozione da Castilho e da altri della sua generazione.

L'opuscolo di C. A. Meschia ${ }^{7}$ è eloquente testimonianza di questa notorietà. Il curatore del libello individua e trascrive ventisette traduzioni dell'ode per un totale di sette lingue (spagnolo, portoghese, inglese, francese, tedesco, catalano e latino ${ }^{8}$ ), ma avverte il lettore che la sua raccolta è tutt'altro che completa, mancando all'appello molte versioni, talune giunte molto tardi, altre promesse e poi mai ricevute, altre non ancora stampate perché sprovviste dei caratteri necessari per la loro impressione. "Tra queste, due traduzioni in armeno [...] e chissà quante me ne saranno scappate!”.

Tra le traduzioni a cui fu soggetta l'ode manzoniana durante il XIX secolo, un numero molto ampio vide come lin-

${ }^{5}$ Alessandro Manzoni, Il cinque maggio, Tutte le poesie (1812-1872), II, Venezia, Marsilio, 1951, vv. 23-24, fonte di tutti i riferimenti.

${ }^{6}$ Vitorino Nemésio, A mocidade de Herculano, p. 286.

${ }^{7}$ Carlo Attilio Meschia, Ventisette traduzioni in varie lingue del "Cinque maggio" di Alessandro Manzoni, Foligno, Campitelli, 1883.

${ }^{8}$ Le traduzioni in latino sono tutte ad opera di autori italiani. 
gua di arrivo proprio il portoghese. Si cimentarono in questa impresa, soprattutto durante la seconda metà del secolo, traduttori professionisti e improvvisati, portoghesi e brasiliani, poeti e prosatori, letterati ma anche diplomatici, medici, storiografi, e persino un sovrano.

José Ramos Coelho (1832-1914) può essere ritenuto il più prolifico tra gli interpreti, con ben dieci edizioni portoghesi del Cinque maggio ${ }^{9}$, che si inscrivono in un arco di tempo compreso tra il 1863 e il 1910 e che riportano sempre qualche modifica, quasi a dimostrare la scrupolosa dedizione con cui l'autore cercò di riprodurre il componimento nella sua lingua natale. A Coelho, che oltre ad essere storiografo, poeta e critico letterario fu anche un affermato traduttore, conosciuto soprattutto per la sua versione della Gerusalemme Liberata, sembra spettasse anche il primato di miglior traspositore dell'ode in lingua portoghese. G. Vegezzi Ruscalla, illustre traduttore della Marília de Dirceu e del Frei Luís de Sousa, scriveva sulla Corrispondenza Letteraria di Torino: "O m'inganno a partito, o quest'ode diventò bilingue" 10 .

La sensibilità linguistica e l'esperienza traduttologica di Ramos Coelho ci permettono inoltre di giustificare l'opinione

${ }^{9}$ In ordine cronologico: Ode a Napoleão, Archivo Pitoresco, 6, 1863 [Lisboa, Typographia Castro Irmão], pp. 310-311; Cinco de Maio, J. Ramos Coelho, Novas poesias, Cruz Coutinho, 1866, pp. 110-117; A morte de Napoleão, Salvador Costanzo, Música Terrenal, Madrid, 1868, pp. 351-352; A morte de Napoleão, Carlo Attilio Meschia, Ventisette traduzioni in varie lingue del "Cinque maggio" di Alessandro Manzoni; A morte de Napoleão, Occidente. Revista Illustrada de Portugal e do Estrangeiro, VIII, 1885 [Lisboa, Empreza do Occidente], p.271; Cinco de Maio. Traducção de J. Ramos Coetho (uma folha solta), Lisboa, Typographia Elzeviriana, 1885; A morte de Napoleão, Joaquim da Silva Mello Guimarães, Ode heróica de Alexandre Manzoni e três versões em portuguez, Rio de Janeiro, Typographia Moreira, Maximino \& C., 1885, pp. 41-48; Cinco de Maio, O Instituto. Revista Scientifica e Litteraria, 34, 1887 [Coimbra, Imprensa da Universidade], pp. 145-147; Cinco de Maio, J. Ramos Coelho, Lampejos, Lisboa, Typographia Castro Irmão, 1896, pp. 93-98; Cinco de Maio, J. Ramos Coelho, Obras poéticas, Lisboa, Typographia Castro Irmão, 1910, pp. 142-143.

${ }^{10}$ J. Ramos Coelho, Lampejos, p. 98. 
ampiamente condivisa secondo cui Il cinque maggio sarebbe un testo quasi intraducibile ${ }^{11}$. Dopo aver passato in rassegna le maggiori difficoltà in cui si è imbattuto, in nota a una delle sue versioni del Cinque maggio, il traduttore conclude:

Todos os que estudaram com certa profundeza [...] as duas línguas, confessam, e não podiam dissimular, vantagens que a italiana leva à nossa. $^{12}$

Con queste parole, Coelho si riferisce alla duttilità, alla vasta gamma di varianti e di libertà che l'italiano offre a chi lo parla o lo scrive. Una caratteristica che rende particolarmente complessa la traduzione di testi italiani di tipo poetico, in cui il problema della conservazione del ritmo, della metrica e della rima dell'originale si somma a quello dell'equivalenza di significato tra parole della lingua di partenza e quelle della lingua di arrivo. Laltro maggiore problema traduttologico individuato da Coelho è invece di tipo stilistico. Un'adeguata resa della sintesi, della rapidità, della nervosa concisione con cui vengono evocate le immagini nella versione originale. Lo stesso lettore potrà apprezzare le soluzioni adottate dal traduttore per ovviare alle difficoltà presentate dal componimento, confrontando in appendice la versione di Ramos Coelho con l'originale manzoniano.

Restando in Portogallo, abbiamo poi la traduzione dello statista nonché poeta e drammaturgo José da Silva Mendes Leal $^{13}$ (1818-1886), che già aveva dato prova di interesse sia per la figura di Napoleone ${ }^{14}$ che per la tematica risorgimen-

\footnotetext{
${ }^{11}$ Nella nota che introduce la traduzione di Ramos Coelho contenuta in Archivo Pitoresco, si legge ad esempio: "Esta poesia, não só é tida e havida pelo melhor trecho lyrico deste século, mas a sua traducção tem intimidado até os engenhos de maior estro", p. 310.

${ }^{12}$ J. Ramos Coelho, Lampejos, p. 97.

${ }^{13}$ José da Silva Mendes Leal, Cinco de Maio, O Instituto. Revista Scientifica e Litteraria, 34, II, 1887[Coimbra, Imprensa da Universidade], pp. 97-100.

${ }^{14}$ J. da Silva Mendes Leal, Napoleão no Kremlin, Lisboa, Typographia da Gazeta de Portugal, 1865.
} 
tale, avendo dedicato un epicedio dai toni eroici al re Carlo Alberto di Savoia ${ }^{15}$.

Portoghese di origini italiane, António José Viale (18061889) nasce a Lisbona da genitori entrambi liguri. Grande erudito latinista ed ellenista, Viale si distinse per le sue traduzioni dal latino, dal greco e dall'italiano al portoghese, tra cui anche la Commedia dantesca. A quanto pare, non possiamo tuttavia estendere tali meriti di traduttore alla sua versione del Cinque maggio ${ }^{16}$ che sembra essere l'unica, tra le versioni portoghesi di cui disponiamo, che non mantiene la disposizione e la qualità delle rime, le parole sdrucciole ed altre caratteristiche formali che rendono il componimento di Manzoni particolare dal punto di vista ritmico. Lo stesso Viale, nel prologo del volume, riconosce la modestia della sua prova, avvertendo il pubblico che il suo unico merito è quello di essere stato scrupolosamente - forse eccessivamente - fedele all'originale.

Ad ogni modo, Il Cinque maggio riscosse un enorme successo non solo in Portogallo ma anche oltreoceano, in Brasile, meritando l'interesse di alcune personalità di spicco che orbitavano attorno alla corte di D. Pedro de Alcântara, secondo e ultimo imperatore del Brasile.

Lo stesso D. Pedro II (1825-1891), amante appassionato della poesia italiana ${ }^{17}$, inviò direttamente ad Alessandro

${ }^{15}$ Vale la pena citare alcuni versi della poesia Ave César, con cui Mendes Leal loda le gesta del re piemontese artefice della prima guerra di indipendenza italiana, per rilevare la patente influenza de Il cinque maggio: "Salve, ó rei! Rei no sólio e no abandono/ mais rei no exílio do que os reis no trono/ Rei até sobre o pó!” (J. da Silva Mendes Leal, Ave César, Cânticos, 1850, pp. 327-332). Si comparino tali versi con l'ode manzoniana Il cinque maggio: "Lui folgorante in solio/ vide il mio genio e tacque/ [...] La fuga e la vittoria/ la reggia e il tristo esiglio/ due volte nella polvere/ due volte sull'altar".

${ }^{16}$ António José Viale, Il cinque maggio. Ode heroica de Alexandre Manzoni traduzida com escrupulosa fidelidade por António José Viale, Coimbra, Imprensa da Universidade, 1885.

${ }^{17}$ Colui che Lamartine soleva chiamare il principe filosofo era nipote da parte di madre del Granduca di Toscana, e il medico della casa imperiale fu nientemeno che 
Manzoni, con cui intratteneva una seppur non molto fitta corrispondenza, la propria traduzione autografa dell'ode accompagnata da una lettera, anch'essa scritta di proprio pugno e datata al 15 Novembre $1871^{18}$. L'anno seguente, a dimostrazione dell'amicizia e della stima reciproca che legava i due, l'imperatore si sarebbe recato a visitare lo scrittore nella sua residenza a Brusuglio. L'ammirazione per Manzoni e l'apprezzamento della sua lirica dovevano di certo essere grandi se il sovrano decise di tradurre un'ode che, di fatto, celebra e omaggia proprio chi, sessantaquattro anni prima, aveva invaso la patria di suo nonno, João VI di Portogallo.

Un altro traduttore di nazionalità brasiliana è Francisco Bonifácio de Abreu (1819-1887). Oltre ad essere parlamentare, poeta e membro di varie società scientifiche e letterarie, Abreu, meglio conosciuto come il barão da Vila da Barra, venne anche nominato colonnello chirurgo onorario dell'esercito imperiale in seguito ai servizi prestati durante la guerra contro il Paraguay. Come D. Pedro II, anch'egli tradusse in portoghese passi della Commedia di Dante e l'ode dedicata a Napoleone ${ }^{19}$.

Luís Vicente de Simoni, uno dei veicoli di conoscenza del Romanticismo italiano a Rio de Janeiro (cfr. Rita Marnoto, "O Ramalhete poético do Parnaso italiano - a Língua portuguesa como arauto da poesia italiana na América do Sul”, Congresso Internazionale il Portogallo e i Mari, un incontro tra culture, a cura di Maria Luisa Cusati, Napoli, IUO, Liguori, 1997). Nel 1843 D. Pedro II aveva sposato la sorella del re Ferdinando II di Napoli nella città partenopea.

${ }^{18} \mathrm{La}$ lettera in questione è contenuta in A. Manzoni, Epistolario. Lettere dal 1854 al 1873, Roma, Biblioteca Italiana, 2008, mentre la traduzione dell'ode di cui ci siamo serviti compare in almeno due edizioni distinte: J. da Silva Mello Guimarães, Ode heróica de Alexandre Manzoni e três versões em portuguez, e Dom Pedro II, Poesias completas com um prefácio de Medeiros e Albuquerque, Rio de Janeiro, Guanabara, 1889.

${ }^{19}$ Il breve opuscolo contenente la traduzione di F. B. de Abreu, reperito presso il fondo manzoniano della Biblioteca Nazionale Braidense, non riporta purtroppo nessuna informazione circa la casa editrice, il luogo e l'anno in cui è venne dato alle stampe. 
Si cimentò nella trasposizione dell'ode anche Francisco Adolfo de Varnhagen ${ }^{20}$ (1816-1878), visconte di Porto Seguro. Passato alla storia come il padre della storiografia moderna brasiliana, Varnhagen fu anche diplomatico e militare; anzi, cominciò la sua carriera militare all'epoca delle guerre liberali proprio come volontario nelle truppe del padre di D. Pedro II, in lotta contro D. Miguel I di Portogallo. Nel 1840 conobbe poi l'imperatore in persona, allora appena quindicenne, con cui intratterrà una duratura amicizia.

Dopo questo breve excursus sul successo riscosso dall'ode e sulle versioni portoghesi di questa che videro la luce nel XIX secolo, viene spontaneo chiedersi quali furono i fattori che in questo periodo contribuirono a un tale interesse sia letterario che traduttologico per la produzione di Manzoni, in particolare per la sua ode Il cinque maggio.

Sfogliando un qualsiasi manuale o dizionario di letteratura portoghese ${ }^{21}$, ci si rende subito conto che Manzoni viene costantemente menzionato, insieme a Foscolo, Leopardi e Pellico, come uno degli autori più tradotti e quindi più letti in Portogallo e Brasile durante il XIX secolo, non solo dal vasto pubblico ma anche da fruitori più specializzati quali il già citato Castilho, ma anche Garrett, Herculano ed altri esponenti del Romanticismo portoghese.

I punti di contatto tra i quattro autori italiani sono abbastanza evidenti. Vissuti a cavallo tra due secoli e due epoche

${ }^{20}$ Francisco Adolfo de Varnhagen, $\grave{A}$ morte de Napoleão, in J. da Silva Mello Guimarães, Ode heróica de Alexandre Manzoni e três versōes em portuguez. Si registra comunque un'edizione anteriore della traduzione, contenuta in José Ferreira Monteiro, Lisia poética. Colleçãao de poesias modernas de autores portuguezes, II, Rio de Janeiro, Typografia Commercial, 1857.

${ }^{21}$ La bibliografia di cui ci siamo serviti è la seguente: Dicionário de literatura portuguesa, ed. Álvaro Manuel Machado, Lisboa, Presença, 1996; Dicionário do Romantismo literário português, ed. Helena Carvalhão Buescu, Lisboa, Caminho, 1997; Teófilo Braga, História do Romantismo em Portugal, Lisboa, Nova Livraria Internacional, 1880; G. Carlo Rossi, A literatura italiana e as literaturas de língua portuguesa, Porto, Telos, 1973. 
storiche, essi non solo assistettero ma parteciparono attivamente ai grandi cambiamenti che tra Settecento e Ottocento erano in atto su più fronti: sul piano letterario, essendo stati tra i primi ad aderire al pensiero e all'estetica romantici; sul piano politico e sociale, sostenendo, chi più, chi meno apertamente, ${ }^{22}$ idee liberali e rivolte alla lotta per la conquista di un'unità e un'indipendenza nazionali.

Sembra essere proprio quest'ultimo aspetto la causa principale dell'enorme interesse letterario e traduttologico di cui fu oggetto Manzoni nel Portogallo e nel Brasile del XIX secolo. In quest'epoca di stravolgimenti sociali e di battaglie a favore della democrazia, la letteratura era considerata innanzitutto uno strumento per dar voce al genio nazionale, ispirato dall'impegno morale e politico. Una concezione tipicamente romantica che troviamo perfettamente sintetizzata in Pinheiro Chagas ${ }^{23}$, per il quale la poesia è l'angelo custode delle nazioni che, sentendo i dolori e le passioni che affliggono gli uomini, scende dal cielo per dettare loro strofe che parlano di libertà, fratellanza, amore per la patria. In Italia, l'angelo consolatore della poesia era sceso per prestare la sua voce a un popolo oppresso dalla tirannia austriaca, a una

Terra che l'oro

porta, costretta, allo straniero, è schiava ${ }^{24}$

${ }^{22}$ L'impegno sociale e il pensiero politico di Manzoni emergono principalmente nelle sue odi civili (Marzo 1821, Il proclama di Rimini, Aprile 1814 e Il cinque maggio), composte tutte nel 1821, un anno segnato dalla morte di Napoleone e dalle insurrezioni piemontesi finalizzate all'ottenimento di una costituzione e dell'indipendenza dall'oppressione austriaca. Il liberalismo manzoniano si presenta tuttavia indissolubilmente legato a una forte componente religiosa e provvidenzialista tutta personale, caratteristica comunque molto apprezzata dal pubblico ottocentesco, che vedeva in questo austero raccoglimento il ritorno a una fede restaurata, più interiore, poetica e idealizzata.

${ }^{23}$ Manuel Joaquim Pinheiro Chagas, Ensaios críticos, Porto, Casa da Viuva Moré, 1866.

${ }^{24}$ Alessando Manzoni, Aprile 1814, Tutte le poesie (1812-1872), vv. 38-39. 
Si capisce quindi quanto il Romanticismo italiano, così strettamente legato all'impegno civile e alle lotte risorgimentali, infervorasse gli animi e destasse un forte entusiasmo al di fuori della penisola.

A corroborare tale tesi, si aggiunga la periodizzazione delle traduzioni dell'ode in lingua portoghese. Pur considerando il lieve ritardo con cui Il cinque maggio fu pubblicato a causa della censura austriaca a cui venne sottoposto, vediamo che la prima traduzione in lingua straniera, ad opera del Goethe, è datata al 1822, appena un anno posteriore alla composizione dell'originale. Le versioni portoghesi di cui abbiamo notizia, al contrario, si inscrivono in un arco compreso tra il 1857 e il 1910, dunque più avanti nel tempo, ciò che potrebbe essere messo in relazione al periodo di sconvolgimenti sociopolitici che vissero sia l'Italia che le due nazioni lusofone, e che portarono da un lato all'unità e alla liberazione del nostro Paese dal giogo austriaco, e dall'altra all'ottenimento di una costituzione liberale e poi di una repubblica.

Riassumendo, il significativo numero di traduzioni del Cinque maggio che si registrano nel Portogallo e nel Brasile del XIX secolo sembrano spia non solo di un generale interesse per la letteratura italiana del tempo, ma anche e soprattutto del fascino esercitato dall'epopea italiana del Risorgimento e dagli scrittori che vi presero parte grazie alla loro fervente attività letteraria. Si spiega quindi perché sia il Manzoni politicamente e socialmente engagé che riconosciamo nelle odi civili ad essere stato tanto letto e tradotto nei due Paesi.

$\mathrm{Al}$ sentimento di ammirazione verso un Paese in lotta per l'indipendenza e l'unità nazionale, si aggiungano poi il mito dell'Italia, penisola soleggiata nonché patria di Dante e di Petrarca, e soprattutto il fascino esercitato dal suo idioma, per la sua musicalità e armoniosità, la lingua "del bel paese là dove 'l sì suona". 


\section{APPENDICE}

Il cinque maggio

(Alessandro Manzoni) $^{25}$

Ei fu. Siccome immobile,

Dato il mortal sospiro,

Stette la spoglia immemore

Orba di tanto spiro,

Così percossa, attonita

La terra al nunzio sta,

Muta pensando all'ultima

Ora dell'uom fatale;

Né sa quando una simile

Orma di piè mortale

La sua cruenta polvere

A calpestar verrà.

Lui folgorante in solio

Vide il mio genio e tacque;

Quando, con vece assidua,

Cadde, risorse e giacque,

Di mille voci al sonito

Mista la sua non ha.

Vergin di servo encomio

E di codardo oltraggio,

Sorge or commosso al subito

Sparir di tanto raggio;

E scioglie all'urna un cantico

Che forse non morrà.

\author{
Cinco de Maio \\ (traduzione di J. Ramos Coelho) ${ }^{26}$
}

Foi; já não é; qual gelido,

Sem voz, sem movimento,

Ficou seu corpo exanime

Orphão de tanto alento,

Assim ferida, attonita,

Co’a nova a terra está,

Muda na hora ultima

Do homem fatal pensando;

Nem sabe se outro egregio

Virá, como elle, e quando

Seu pó, de sangue humido,

Como elle, pizará.

Brilhante o viu no solio

$\mathrm{O}$ genio meu; cahido

Depoi; depois no imperio;

Depois emfim vencido;

E do universo ao fremito

Sua voz unir não fez.

Virgem de servo encomio

E de covarde insulto,

Acorda ao sol esplendido,

Tão de repente occulto,

E solta á morte um cantico,

Que é do porvir talvez.

25 Tutte le poesie (1812-1872).

${ }^{26}$ Si riporta qui una delle versioni ad opera di J. Ramos Coelho. Cfr. J. Ramos Coelho, Lampejos, Lisboa, Typographia Castro Irmão, 1896, pp. 93-98. Punteggiatura, diacritici e grafia appartengono all'originale. 
Dall'Alpi alle Piramidi,

Dal Manzanarre al Reno,

Di quel securo il fulmine

Tenea dietro al baleno;

Scoppiò da Scilla al Tanai,

Dall'uno all'altro mar.

Fu vera gloria? Ai posteri

L'ardua sentenza: nui

Chiniam la fronte al Massimo

Fattor, che volle in lui

Del creator suo spirito

Più vasta orma stampar.

La procellosa e trepida

Gioia d'un gran disegno,

L'ansia d'un cor che indocile

Serve, pensando al regno;

$E$ il giunge, e tiene un premio

Ch'era follia sperar;

Tutto ei provò: la gloria

Maggior dopo il periglio,

La fuga e la vittoria,

La reggia e il tristo esiglio;

Due volte nella polvere,

Due volte sull'altar.

Ei si nomò: due secoli,

L'un contro l'altro armato,

Sommessi a lui si volsero,

Come aspettando il fato;

Ei fe'silenzio, ed arbitro

S'assise in mezzo a lor.

E sparve, e i dì nell'ozio

Chiuse in sì breve sponda,

Segno d'immensa invidia
Dos Alpes ás Pyramides, Do Rheno ao Manzanares, Raio, o veloz relampago Seguiu, rasgando os ares; Troou de Scylla ao Tanais, De um mar a outro mar.

Foi verdadeira gloria? Aos tempos a sentença; Nos do Senhor curvemo-nos Á potestade immensa, Que n'elle quiz a maxima Sua obra apresentar.

O procelloso e trepido Prazer de uma alta empreza, A ancia de um peito indomito Que sonha a realeza, $\mathrm{E}$ a ganha, e alcança um premio Que era loucura esp'rar.

Tudo provou; a gloria Maior depois do p'rigo, A fuga e a victoria, O throno e o exilio imigo. No pó duas vezes, prospero Duas vezes sobre o altar.

Appareceu; dois seculos, Um contra o outro armado, Para elle olharam timidos, Como aguardando o fado; Calae-vos disse; e arbitro Entre ambos se foi pôr.

Despareceu; e em ocio, Findou, longe do mundo, N'uma ilha, alvo continuo 
E di pietà profonda,

D'inestinguibil odio

E d'indomato amor.

Come sul capo al naufrago

L'onda s'avvolve e pesa,

Londa su cui del misero,

Alta pur dianzi e tesa,

Scorrea la vista a scernere

Prode remote invan;

Tal su quell'alma il cumulo

Delle memorie scese!

Oh quante volte ai posteri

Narrar se stesso imprese,

E sull'eterne pagine

Cadde la stanca man!

Oh quante volte, al tacito

Morir d'un giorno inerte,

Chinati i rai fulminei,

Le braccia al sen conserte,

Stette, e dei dì che furono

L'assalse il sovvenir!

E ripensò le mobili

Tende, e i percossi valli,

E il lampo de' manipoli,

E l'onda dei cavalli,

E il concitato imperio

E il celere ubbidir.

Ahi! forse a tanto strazio

Cadde lo spirto anelo,

E disperò; ma valida

Venne una man dal cielo,

$\mathrm{E}$ in più spirabil aere

Pietosa il trasportò;
Da inveja e dó profundo,

De inextinguivel odio,

$\mathrm{E}$ de indomado amor.

Qual sobre a fronte ao naufrago

Se enrola e cae pesada

A vaga, d'onde o misero,

Co'a vista, alta, alongada,

Buscava emtorno, ávido,

Praia longinqua em vão,

Tal n'aquell'alma o cumulo

Tombou de mil memorias.

Oh! quanta vez aos posteros

Tentou narrar suas glorias,

E nas eternas paginas

Cahiu sem força a mão!

Oh ! quantas no fim tacito

De um dia sem proveito,

No chão o olhar fulmineo,

Os braços sobre o peito,

Inteiro o seu preterito

Viu de repente erguer.

Lembrou as tendas moveis,

O acometter dos vallos,

Do aço o brilho tremulo,

As ondas dos cavallos,

E o concitado imperio,

E o prompto obedecer.

Ai! á tamanha magoa

Cedeu talvez afflicto

e desesp'rou; mas valido

Braço desceu bemdicto,

E para outro ar mais limpido

Piedoso o transportou; 
E l'avviò, pei floridi

Sentier della speranza,

Ai campi eterni, al premio

Che i desideri avanza,

Dov'è silenzio e tenebre

La gloria che passò.

Bella immortal! benefica

Fede ai trionfi avvezza!

Scrivi ancor questo, allegrati;

Che più superba altezza

Al disonor del Golgota

Giammai non si chinò.

Tu dalle stanche ceneri Sperdi ogni ria parola:

II Dio che atterra e suscita,

Che affanna e che consola, Sulla deserta coltrice

Accanto a lui posò.
E pelas sendas floridas

O conduziu da esp'rança

Ao campo eterno, ao premio

Que mais que o anhelo alcança,

Onde é negror, silencio

A gloria que passou.

Fé immortal, benefica.

De palmas bella e ufana,

Colhe mais esta; alegra-te,

Que nunca outra mundana

Grandeza egual do Golgotha

Á affronta se humilhou;

Exulta; e o resto inanime

Preserva da maldade;

Quem mata e abre os tumulos, Quem pune e tem piedade, Deus, no seu leito funebre

Ao pé se lhe assentou. 